\title{
Connaître le bénéfice social pour mieux évaluer la hausse des coûts
}

\author{
Nora Willea, Jürg Schlup ${ }^{b}$ \\ a Dr phil., Collaboratrice scientifique du président, ${ }^{\mathrm{b}} \mathrm{Dr}$ méd., président de la FMH
}

La politique de santé ne se contente plus de déterminer quelles améliorations pourraient amener à un système de santé moins cher. De plus en plus, elle vise aussi à limiter les prestations. Cette tendance est favorisée par le fait que, pour de nombreuses personnes, les bénéfices de la prise en charge médicale pour les patients sont moins visibles que les chiffres inquiétants des coûts. Cependant, c'est le fait que la hausse des coûts s'accompagne d'un bénéfice social qui mérite toute notre attention.

Aristote déjà a évoqué la difficulté de décider de quel côté la balance devait pencher-la santé ou les dépenses qu'elle occasionne: «Souvent, le même homme change d'avis à ce sujet: malade, il place le bonheur dans la santé, et pauvre, dans la richesse» [1]. Il est encore plus difficile d'arbitrer entre l'argent et la santé lorsque l'on dispose d'une seule source d'information: tandis que les coûts sont clairement chiffrables, la notion de "santé» est souvent moins nette. Qu'obtient-on en retour des dépenses consenties? Quels bénéfices sont visés? La hausse des dépenses apporte-t-elle aujourd'hui un bénéfice social équivalent?

La question des coûts est indiscutable. Lorsque la LAMal a été introduite en 1996, l'ensemble des coûts du système de santé suisse s'élevait à 39,6 milliards de francs, soit $9,7 \%$ du PIB, pour une population moyenne de 7,1 millions d'habitants [2]. Les derniers chiffres de l'OFS montrent que notre système de santé coûte au total 74,6 milliards de francs pour 8,2 millions de personnes, ce qui représente $11,6 \%$ du PIB [2]. Cette hausse des coûts que l'on observe également dans d'autres pays de l'OCDE vient directement peser sur le porte-monnaie des assurés. Tandis que l'ensemble des coûts de santé n'ont augmenté «que» de 72\% depuis 1996 (hors renchérissement), la prime moyenne de l'AOS a augmenté de $107 \%$ [3].

Cette hausse des coûts est due à deux raisons principales: le progrès médical et le vieillissement de notre société. Appelés communément «inducteurs de coûts», ces deux facteurs pourraient être rebaptisés «indicateurs de bénéfice» compte tenu des évolutions très positives qu'ils expriment.

\section{Depuis 1996, le nombre d'années de vie potentiellement perdues a diminué de $40 \%$}

Même si l'allongement de l'espérance de vie ne peut être attribué qu'à la qualité des soins de santé, il est malgré tout impressionnant de constater que les hommes et les femmes en Suisse ont vécu en 2015 respectivement 4,7 et 2,9 années de plus qu'en 1996 [4]. Et cette évolution ne profite pas uniquement à la catégorie des personnes très âgées: les décès prématurés, sur la base du nombre d'années de vie perdues avant 75 ans, ont aussi nettement reculé. Tandis qu'en 1996, on enregistrait encore 5318 années potentielles de vie perdues par 100000 habitants, ce chiffre ne s'élevait plus qu'à 3200 en 2014 [5].

Ce gain de durée de vie est également dû au progrès médical, comme le montrent par exemple les évolutions pour les maladies cardiovasculaires et les cancers, soit les deux groupes de maladies responsables de la plupart des décès [6] ou des années de vie perdues en Suisse. [5] Le nombre de nouveaux cas de cancer est passé d'environ 30000 en 1996 à quelque 42000 en 2015 [7] . Cette augmentation s'explique par le fait que notre population a non seulement augmenté, mais surtout vieilli, l'âge constituant un facteur de risque important pour le cancer. Parallèlement, on observe un net recul des taux de mortalité, grâce aux diagnostics précoces et aux nouvelles méthodes thérapeutiques. Ainsi, le nombre de personnes survivant au diagnostic du cancer ne cesse de croître: en 2015, la Suisse comptait environ 120000 personnes ayant survécu au cancer depuis dix ans, soit deux fois plus qu'en 2000 [7]. 
Le nombre d'hospitalisations liées aux maladies cardiovasculaires a augmenté de $20 \%$ entre 2000 et 2014 en raison de l'augmentation mais aussi du vieillissement de la population [8]. Là encore, le chiffre des décès dus à ces maladies a diminué en absolu de $12 \%$ sur cette même période [8]. En 2004, par exemple, 23\% des patients hospitalisés pour un infarctus du myocarde sont décédés tandis qu'ils n'étaient "plus que» $14 \%$ dix ans plus tard [5]. Le taux de mortalité des patients souffrant d'insuffisance cardiaque est également tombé de 35 à $22 \%$ entre 2004 et 2014 et, pour les AVC, on observe sur la même période un recul de la mortalité de 24 à 17\% [5].

\section{De meilleurs traitements améliorent la qualité et l'espérance de vie, mais accroissent aussi la demande}

De tels succès thérapeutiques sont toutefois susceptibles d'accroître considérablement la demande de prestations médicales. Contrairement à un patient décédé, le patient ayant survécu sollicite des prestations de réadaptation, prend régulièrement des médicaments, consulte son médecin et tombera à nouveau malade quelques années plus tard. L'amélioration des soins médicaux génère souvent aussi une plus grande demande pour les cas de maladies non létales: de plus en plus de patients peuvent envisager une opération de la cataracte, du canal carpien, des hémorroïdes ou de problèmes cardiaques, car les progrès de la médecine ont permis entre autres de diminuer les risques et les effets secondaires [9].

Ces quelques exemples permettent d'illustrer la question du volume des prestations et des bénéfices pour les patients. L'avantage des soins de qualité ne se limite cependant pas à l'espérance ou à la meilleure qualité de vie dont le patient et ses proches vont profiter. Des traitements réussis ont également une portée économique, puisqu'ils évitent ou réduisent les arrêts de travail, empêchent les départs en préretraite, retardent ou évitent l'invalidité et la dépendance aux soins. Pour de nom- breuses maladies fréquentes, les coûts indirects liés à la perte de productivité et la dépendance aux soins sont plus élevés que les dépenses des traitements ambulatoires, hospitaliers et médicamenteux. Ces coûts indirects représentent $73 \%$ et $60 \%$ des coûts globaux respectivement des troubles psychiques et des cas de diabète [3]. D'un point de vue économique, des dépenses plus élevées en médecine peuvent donc contribuer à économiser dès lors qu'elles réduisent les coûts indirects de la santé.

Les avantages apportés ces dernières décennies par les progrès de la médecine sont tangibles de la néonatologie et l'obstétrique à la gériatrie et la médecine palliative, et profitent à l'ensemble de la population. Les exemples présentés dans les prochaines éditions du BMS par les représentants de plusieurs spécialités constitueront un précieux complément à la discussion sur la politique de santé: pour déterminer l'ampleur des soins de santé que nous voulons et à quel prix, nous devons prendre conscience, au-delà des coûts, de ce que nous gagnons ou de ce à quoi nous devrions éventuellement renoncer, et si un tel renoncement ne reviendrait pas finalement plus cher.

\section{Références}

1 Aristote. Ethique à Nicomaque.

2 Données de l'Office fédéral de la statistique; Coûts et financement du système de santé depuis 1960 (T 14.5.1.1), publié le 27.04.2017, numéro OFS je-f-14.05.01.01.

3 Interpharma (2017). Santé publique en Suisse. $36^{\mathrm{e}}$ édition.

4 Office fédéral de la statistique, Indicateurs de mortalité en Suisse, 1970-2015, publié le 29.09.2016; numéro OFS su-f-01.04.02.02.01.

5 Site de l'Observatoire suisse de la santé; Indicateurs sur l'état de santé de la population [en date du 26.6.2016]. http://www.obsan. admin.ch/fr/indicateurs.

6 OFS Actualités. Statistique des causes de décès. La mortalité en Suisse et les principales de causes de décès en 2014. Neuchâtel, février 2017.

7 Heusser R, Noseda G. Le cancer en Suisse, rapport 2015: présentation de résultats choisis. Bulletin suisse du cancer 2/2016; 168-72.

8 Site de l'Office fédéral de la statistique; Maladies cardiovasculaires; URL: https://www.bfs.admin.ch/bfs/fr/home/statistiques/sante/ etat-sante/maladies/cardiovasculaires.html (en date du 26.6.2017)

9 Roth S, Pellegrini S. Virage ambulatoire. Transfert ou expansion de l'offre de soins? Obsan Rapport 68; 2015. 